International Journal of Wireless \& Mobile Networks (IJWMN) Vol. 3, No. 6, December 2011

\title{
Performance Evaluation of MAC PROTOCOLS FOR AD-HOC NETWORKS USING DiRECTIONAL ANTENNA
}

\author{
Arvind Kumar $^{1}$, Rajeev Tripathi ${ }^{2}$ \\ Department of Electronics and Communication Engineering, MNNIT, Allahabad \\ arvindk@mnnit.ac.in ${ }^{1}$, remnnit.ac.in ${ }^{2}$
}

\begin{abstract}
An Ad-hoc network is a dynamic network farmed on demand by a group of nodes without any pre-existing network infrastructure. Self configurability and easy deployment feature of the Mobile Ad-hoc network (MANET) resulted in numerous applications in modern era. An efficient and effective medium access control (MAC) protocol is essential in ad-hoc network for proper sharing of channel. The omnidirectional antenna has been used in traditional MAC protocols. More intensive attention is given toward the directional antenna by researchers due to capability of spatial reuse and other beneficial features. Main focuses of this paper is to discus and evaluate the performance of MAC protocol using omni-directional and directional antenna. We conducted a comparative simulation study of different MAC protocols using Omni-directional and directional antenna. Performance metrics like throughput and delay are used for the performance analysis. On the basis of result derived from simulation a comparison among these MAC protocols using directional antenna is given.
\end{abstract}

\section{Keywords}

Ad-hoc network, Directional antenna, Directional MAC protocol, Smart antenna.

\section{INTRODUCTION}

There are two possibilities for enabling wireless communications: infrastructure mode and ad hoc mode. The first one relies on infrastructure that needs to be built in advance. In 802.11 infrastructure mode, all the wireless devices in the network can communicate with each other through an Access Point (AP) or communicate with a wired network as long as the AP is connected to a wired network. The other choice is ad hoc mode whose major feature is the nonexistence of supporting infrastructure. An ad-hoc network is self organized wireless network without fixed or backbone infrastructure. Ad-hoc network topology is changes dynamically due to mobile nature of node hence routing protocols are required for data communication between source and destination. All nodes have routing capability and use peer-to-peer packet transmission or forward packet for other node using multi-hop communication. Due to flexibility in deployment, ad-hoc networks are very useful in military and other application such as emergency and rescue operation where infrastructure is unavailable or unreliable. Wireless mesh networks could be considered as a type of wireless ad hoc networks. Mesh networks extend the reach of wireless networks and are ideally suited for many environments such as commercial zones, neighbourhood communities and university campuses. Wireless sensor networks are another application of ad hoc networks in which sensor devices are connected in open peer-to-peer ad hoc network architecture to offer various utilizations such as monitoring traffic congestion in a city, detecting a biological weapon in the battle field and border intrusion.

Traditional work on ad hoc networks assumes that each device is equipped with omnidirectional antennas. With the continuing reductions in the size and cost of directional antenna 
in recent years, it has become feasible to use directional antennas for ad hoc networks. Directional antenna offer potential benefits for wireless ad-hoc networks. With directional transmission and reception, spatial reuse ratio and antenna gain can be increased substantially; this leads to significant improvement in communication system performance. To best utilize the directional antenna, a suitable MAC protocol is required. Directional antenna based MAC protocols are capable of transmitting only in certain narrow azimuth that significantly reduces the chance of collision and increase the effective network capacity. However, use of directional antenna introduce the some complex issues like hidden terminal, exposed terminal and deafness problem at the same time trans-receiver complexity increases.

Carrier sense medium access (CSMA) [1] protocol causes hidden terminal and exposed terminal problems. These problems are minimized by MACA (medium access collision avoidance) [2] using RTS/CTS (request to send/clear to send) frames. Some optional controls are added for better performance in Multiple Access with Collision Avoidance for Wireless (MACAW)[3]. IEEE 802.11 MAC DCF protocol [4] is CSMA/CA (Carrier sense multiple access with collision avoidance) with optional RTS/CTS control message. The IEEE 802.11 MAC protocol is designed to exploit omni-directional antennas and could not work well in directional antenna based ad hoc networks. Therefore, several modified MAC protocols have been proposed to exploit directional antennas, enhance the spatial reuse, and increase network capacity.

This paper is organized as follows: In Section II brief description of related works already done in area of MAC protocol using directional antenna is given. Section III describes the basic concepts, operation and type of smart antenna. Architecture and operation of MAC protocol is given in Sections IV. Performance evaluation is given in Section V that discusses the simulation setup and simulation result under the static and dynamic topology. Finally, we conclude with future scope of works in Section V.

\section{RELATED WORKS}

In this Section we review some directional MAC protocols proposed in literature for ad-hoc networks using directional antenna. The major advantage of directional antenna with 802.11 based ad-hoc network is the reduced interference and the possibility of having parallel transmission to increase the spatial reuse of radio resources [5]. A number of directional protocols have been proposed for wireless ad-hoc networks using directional antenna.

IEEE 802.11 DCF MAC [6] is originally designed for omni-direction transmission using omnidirectional antenna known as CSMA/CA. If mobile node senses the channel is free/ idle it waits for duration DIFS, if channel is still idle it will wait for additional random duration of time called back off time. Once back off time expires, data transmission is initiated. After each collision $\mathrm{CW}$ (Contention window) size is doubled up to maximum window size. On the other hand after each successful transmission minimum size of window is set. If data packet size is larger than specific threshold, it will use four ways hand shake mechanism for data transmission. If mobile node has packets to transmit, it will transmit the RTS frame (RTS frame having the identification of intended receiver), in reply of RTS receiver will transmit CTS frame. After receiving the CTS frame source will send the DATA packet, after successful reception of DATA, receiver will send the ACK frame for each data packets. These control packet (RTS, CTS) decrease the probability of data collision, as they allow communicating nodes to reserve the channel for the entire communication duration before the actual data transmission begins. All neighbours nodes of sender or receiver are expected to keep silent to avoid collision or interference with ongoing transmission, which causes low spatial reuse. The basic operations of these schemes are illustrated in figures 1(a) and (b). 
International Journal of Wireless \& Mobile Networks (IJWMN) Vol. 3, No. 6, December 2011

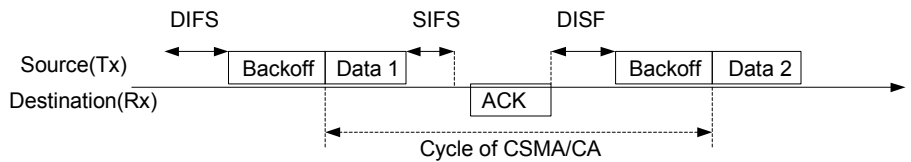

(a)

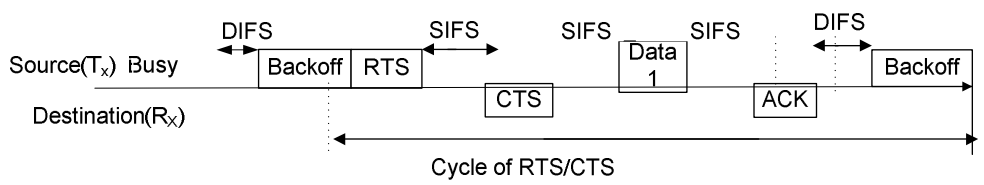

(b)

Figure 1. Timing diagram of (a) CSMA/CA and (b) RTS/CTS

In [7] authors proposed a MAC protocol using directional antenna. It has been assumed that each node knows it own position and neighbour's node, and directional and onmi-directional transmission range are same. Two schemes: directional RTS (DRTS) MAC and omnidirectional RTS (ORTS) MAC have been proposed. Node will send the ORTS frame onmidirectionally if none of the directional antenna elements are blocked, otherwise node will send a DRTS frame, provided that desired directional antenna element is not blocked. In both scheme the DATA/ACK are transmitted directionally, while the CTS is transmitted omni-directionally, if it does not interfere with other ongoing transmission. Nodes have different transmission range for directional and omni-directional transmission in MAC protocol proposed in [8]. All frames are transmitted directionally. RTS frame send using multi-hop while CTS, DATA and ACK are transmitted using single hop. In [9] authors proposed an algorithm in which RTS, CTS, DATA and ACK frames are transmitted using the directional antenna in the directions which are free according to the directional network allocation vector (D-NAV) table. [10] Presents a MAC protocol using omni-directional RTS/CTS and directional DATA, to reduce the level of interference. In this scheme mobile node do not have any location information.

In [11] authors have proposed dual busy tone multiple accesses (DBTMA) and its directional version (DBTMA/DA) in [12]. It divides the channel in two sub channel. Busy tone is transmitted omni-directionally in [11] while directionally in [12]. Authors have split the channel in two sub channels [13], one is used for RTS/CTS/DATA/ACK and the other is used for busy tone transmission. A Selective Directional MAC (SDMAC) protocol is proposed by authors in [14]. This paper deal with the problems, like deafness problem, hidden terminal problem, and Heal on line (HOL) blocking problem. A scheduling algorithm has been proposed to deal with HOL blocking problem. A range adaptive directional MAC is proposed in [15]. In the place of single fold directional transmission range they proposed multi fold transmission range. A range based DNAV and distance between source and destination is used to select the transmission range. In [16] authors give the comparative study of different omni-directional and directional MAC protocols like 802.11 MAC, MACA, MACAW, FAMA, DBTMA and DBTMA/DA.

\section{SMART ANTENNA}

The improvement of the spectral efficiency in Ad-hoc network may be achieved through the application of directional antennas. The employment of directional antennas has demonstrated improvement in the performance of Ad-hoc network. This enhancement is because directional antennas concentrate the power into limited regions, and consequently, they considerably diminish the interference caused to users that are not within these regions. However, directional antennas lack of the required flexibility that ad-hoc networks demand. Automatically reconfiguration of the network is violated by the employment of directional antennas. A more 
appropriate kind of antennas that have the advantages of directional antennas and offer a major flexibility is the so-called smart antennas. Smart antennas generally combine multiple antenna elements with a signal processing capability to optimize its radiation and/or reception pattern automatically in response to the signal environment. Basically, there are two major categories of smart antennas.

\subsection{Type of Smart antenna}

\subsubsection{Switched beam antenna}

Switched beam antenna is comprised of multiple fixed beams that are formed by shifting the phase of each antenna element of an antenna array by a predetermined amount, or simply by switching between several fixed directional antennas. The transceiver can select one or more beams to transmit or receive.

\subsubsection{Adaptive arrays antenna}

Adaptive arrays antenna is theoretically able to form an infinite number of radiation patterns. The patterns are created taking into account the desired signal and the interferers. In other words, they have the capability of direct the main beam toward the desired signal while suppressing the antenna pattern in the direction of the interferers. Adaptive arrays tend to perform better than switched beam antennas, since they place the desired signal at the maximum of the main lobe and reject the interferers. Nevertheless, adaptive arrays are not convenient for ad-hoc networks due to their high complexity and cost. Switched beam antennas, although not performing at the same level of adaptive arrays, offer the advantages of directional antennas joint to a major flexibility, and a lower cost and complexity compared to adaptive arrays.

\subsection{Need of Smart Antenna}

There are many motivations to utilize the smart antenna technique in a wireless system. As an example of a cellular communication system where the capacity has become a critical issue, the use of conventional omni-directional antennas not only causes huge waste of signal energy because only a small part is transmitted to desired receiver but also generates serious interference to neighbouring base stations and terminals. Therefore, dividing one cell into several sectors and uses a directional antenna for transmission was developed with the goal of reducing the interference level. Dividing in sectors has shown ability to increase frequency spectrum utilization. However, sectorized systems lack the ability to change the antenna's beamwidth or orientation in response to a changing propagation environment and traffic condition. This shortcoming results in large capacity waste in sparse traffic sectors and traffic blocks in dense traffic sectors. The adaptive array smart antenna system which can intelligently control its radiation pattern based on signal processing provides an excellent solution to these problems. Its feature of generating null towards interferers results in higher frequency spectrum utilization and thus increases the system capacity.

The smart antenna technique is applicable for almost all major wireless protocols and industrial standards to achieve larger network coverage and higher system capacity. Examples of these standards could be FDMA (frequency division multiple access) employed in AMPS( advanced mobile phone system) and NMT (Nordic mobile Telephone); TDMA( time division multiple access) employed in GSM (global system for mobile communication) and IS-136; CDMA (code division multiple access) employed in IS-95, WCDMA (wideband CDMA) and TDSCDMA(time division synchronous CDMA ); FDD(frequency division duplexing) and TDD (time division duplexing). As its costs continue to decline, the smart antenna offers a practical, economical solution to address wireless network capacity and performance challenges for different communication systems, including RFID, WiMax, Ultra wideband (UWB), and even WiFi. 


\subsection{Antenna model used for simulation}

In this paper we used the switched beam antenna. Each node have $\mathrm{N}$ highly directional, fixed predefined antenna beam elements which are deployed into non overlapping fixed sectors, each spanning an angle of 360/N degree. Each node can transmit in two modes: omni-directional and directional mode. That has been presented in figure. 2 by unicast and broadcast transmission.

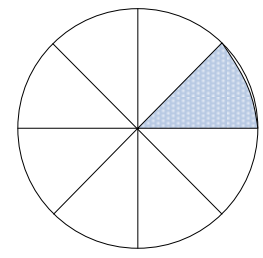

(a)

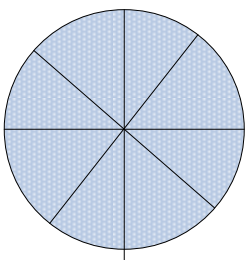

(b)

Figure 2. Azimuth pattern of 8 sector directional antenna for (a) unicast and (b) multicast

When a node $\mathrm{n}$ forms a transmission beam at an angle $\theta$ and a beam width $\Phi$ with a transmission range $R$, the coverage range of node $\mathrm{n}$ at an angle $\theta$ is defined as transmission_zone $\mathrm{n}_{\mathrm{n}}(\theta, \Phi, R)$ as shown in Fig. 3.

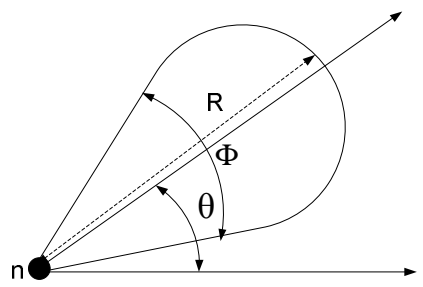

Figure 3. Transmission Zone of a node $\mathrm{n}$ ( $\operatorname{transmission} \_z o n e_{\mathrm{n}}(\theta, \Phi, R)$ ), with beam width $\Phi$, beam angle $\theta$ and transmission range $R$

Without loss of generality, we assume that the direction of each beam is fixed and the boresights of the first sector are always directed towards the $0^{\circ}$ and $\Phi$ on a polar plane, in this case $\theta$ will be $0^{\circ}$. When a link needs to be established for communication between nodes $i$ and $j$, then node $i$ calculates the relative angle, $\phi_{i j}$, between the $0^{\circ}$ in the polar plane and link $(i, j)$ to determine the employed antenna beam. Based on the above assumptions, the selected beam $\xi_{i j}$ that node $i$ would use to communicate with node $j$ via link $(i, j)$ is given by equation given below.

$$
\xi_{i j=}\left\lceil\frac{\phi_{i j}}{\Phi}\right\rceil
$$

Node $j$ will apply the exact same procedure to determine the beam $\phi_{j i}$. Figure 4 shows the distribution of the antenna beams and the beam selected by node $i$ to establish link $(i, j)$.

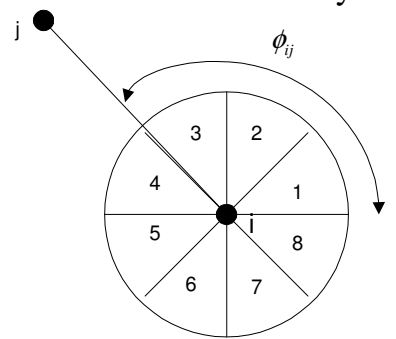

Figure 4. The selection of the beam that is used for link $(i, j)$ is based on angle $\phi_{i j}$ which depict which beam will be used for this communication link. 


\section{Mac Protocol}

Despite recent advances in MANET, there is still a wide range of different open research issues. One of the most important building blocks of wireless ad-hoc networks consists in designing an efficient medium access control (MAC) scheme. A well designed MAC protocol is essential to maximize the performance and the efficiency of the network. One approach for multiple accesses is to employ contention based schemes where nodes compete for accessing the channel. However, contention-based medium access method are inherently inappropriate for providing QoS guarantees, which is becoming a basic premise for lots of communication. Collision-free access techniques are another kind of MAC scheme more suitable for QoS wireless ad-hoc networks, since it is possible to guarantee QoS. All above discussed MAC protocols are based on using omni-directional antenna.

Directional antenna has been broadly used in various communication systems. However, to simply use directional antenna with the conventional IEEE 802.11 standard for ad hoc network could not bring substantial network improvements. This encourages many researchers to develop new MAC protocols which could fully exploit the advantages of directional antennas.

\subsection{MAC layer problems using directional transmission}

The protocols mentioned in introduction sections allow the spatial reuse and performance enhancement. However, these methods fails to address the issues arise using the directional transmission, like deafness problem, hidden terminal problem and HOL blocking problem.

\subsubsection{Deafness problem}

Deafness occurs when a node attempts to transmit to a node, which is already busy in transmission in another direction [7]. Deafness is due to mainly directional RTS/CTS messages. The destination node using a directional antenna is deaf in all directions except for the direction of transmission/reception; such a node does not receive, and hence, does not respond to any RTS requests from other directions. In most cases, the attempting node is also deaf to the destination's transmission/reception, since it cannot sense the ongoing directional communication. In the literature, some solutions are proposed to alleviate the deafness problem with directional transmissions. Sub-band busy tone [8] and circular MAC [10] prevent from defenses problem in Ad-hoc network using directional antenna.

\subsubsection{Hidden terminal problem}

A hidden terminal can be defined as a terminal that is not aware of the ongoing communication between transmitter/receiver pairs and whose intended transmission could lead to the failure of the ongoing transmitter/receiver pair's communication. Conventional MAC protocols for ad hoc networks (the IEEE 802.11 operated in ad hoc mode using -directional antenna) address the hidden terminal problem by employing the RTS/CTS handshaking mechanism before data transmission. Using directional antenna at physical layer, RTS/CTS packets are transmitted directionally. Using directional antenna hidden terminal problem is due to unheard RTS/CTS. Hidden terminal problem results from the feature of a directional antenna that its antenna gain towards a desired direction is larger than the gain towards other directions. Multiple access and busy tone solution are presented in [1].

\subsubsection{HOL (Head of Line Blocking) problem}

This effect occurs because it is possible for the medium to be free in some directions but not others. Head of Line blocking phenomenon is common in First in First Out (FIFO) nature queues. This problem becomes significant in wireless ad hoc networks using directional antennas as it uses FIFO queue which consists of packets intended for different directions. A 
packet on the top of the queue may block the remaining packets if it finds the medium busy in its intended direction, where as the packets in the queue intended for other directions may find the medium to be idle. This problem reduces the performance of the protocol to a great extent.

\subsection{Architecture}

This section details the architecture and operation of MAC protocol. In this architecture every node keeps a MAC table that gives the blocked and unblocked beams and best beam for transmission between source and destination. All nodes send and receive the packet directionally. We assume every node know the relative direction of its neighbouring nodes. This information can achieved through GPS system installed at each node. Switching between beams and blocking of the beams are done via radio frequency switches. At the receiving node selective diversity is applied to determine the best beam over which the highest SNR is measured. Beam information is receded and updated during the RTS and CTS frame exchange, frame format are depicted in figure 4.

RTS/CTS frame of given MAC protocol has one and three extra fields than the frame defined in IEEE802.11 MAC protocol. "Beam Number" is used to indicate the beam number used by the transmitter to send RTS/CTS packets. "Best beam number" indicate the best beam on the basis of SNR to be used by the transmitter and receiver. Other fields have the same meaning as in IEEE802.11 MAC frame. This concept had been given in [20] and used for wireless mesh networks but with four antenna beams. We have used this concept with eight antenna beams to improve the MAC performance when number of node is large which is presented as AngularMAC with eight beams "A-MACEB".

\begin{tabular}{|c|l|c|c|c|l|}
\hline $\begin{array}{c}\text { Frame } \\
\text { Control }\end{array}$ & Duration & $\begin{array}{c}\text { Receiver } \\
\text { Address }\end{array}$ & $\begin{array}{c}\text { Transmitter } \\
\text { Address }\end{array}$ & $\begin{array}{c}\text { Transmitter } \\
\text { Beam Status }\end{array}$ & FCS \\
\hline
\end{tabular}

(a)

\begin{tabular}{|c|c|c|c|c|c|c|c|}
\hline $\begin{array}{c}\text { Frame } \\
\text { Control }\end{array}$ & Duration & $\begin{array}{c}\text { Receiver } \\
\text { Address }\end{array}$ & $\begin{array}{c}\text { Transmitter } \\
\text { Address }\end{array}$ & $\begin{array}{c}\text { Transmitter } \\
\text { Beam Status }\end{array}$ & $\begin{array}{c}\text { Receiver } \\
\text { Best Beam }\end{array}$ & $\begin{array}{c}\text { Transmitter } \\
\text { Best Beam }\end{array}$ & FCS \\
\hline
\end{tabular}

(b)

Figure 4. (a) RTS and (b) CTS frame format

\subsection{MAC Operation}

When node $\mathrm{A}$ has data for transmission to node $\mathrm{B}$, if it senses channel is free, then it will transmit RTS frame using all beams. RTS [B, A, n] packet are sent over all beam and they are received over the beams of surrounding nodes as shown in figure 5 . Where $n$ is used to represent the 8 beam starting from 1 to 8 and 8 bits are being used and bit position value in bit pattern represent the each beam. Bit pattern 00000100 represent that this packet is being transmitted using beam number 3 (3rd bit is 1). RST [B, A, 8] indicate that RST packet is transmitted to node $\mathrm{B}$ using beam 8 of node $\mathrm{A}$ and it is being received by the node $\mathrm{B}$ over beam 5. Other RTS transmitted by the beams of $\mathrm{A}$, may be received by the node $\mathrm{B}$, but on the basis of SNR value beam 5 is selected. Node $\mathrm{C}$ and $\mathrm{D}$ also receive the RTS packet transmitted because they are in transmission range of A but they will not reply. $\mathrm{C}$ and $\mathrm{D}$ record the beam number used by $\mathrm{A}$ as best beam for communication to $A$ that can be used in future. After receiving the RTS intended to $\mathrm{B}$, it will sent CTS packet using all beams. CTS $[\mathrm{A}, \mathrm{B}, 4,8,4]$ indicate that $\mathrm{B}$ is source and $\mathrm{A}$ is destination of CTS packet, B use the beam number 4 , beam number 8 and 4 are best beam of node $\mathrm{A}$ and $\mathrm{B}$ respectively for transmission between $\mathrm{A}$ and $\mathrm{B}$. CTS [A, B, n, 8, 4] are sent by all beam of node $B$ to its surrounding. Node $C$ also receives the CTS [A, B, 4, 8, 4] over its beam number 7. Node $\mathrm{C}$ will block its beam 4 and 7 for transmission between $\mathrm{A}$ and $\mathrm{B}$ because 


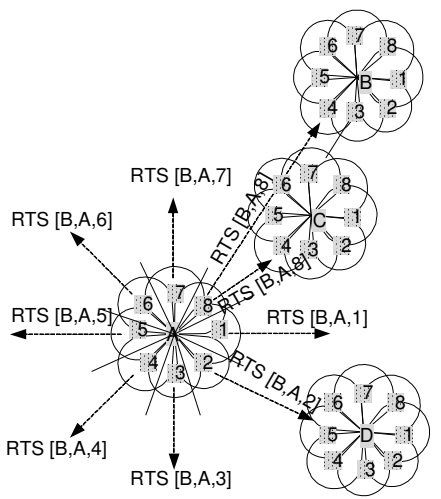

(a)

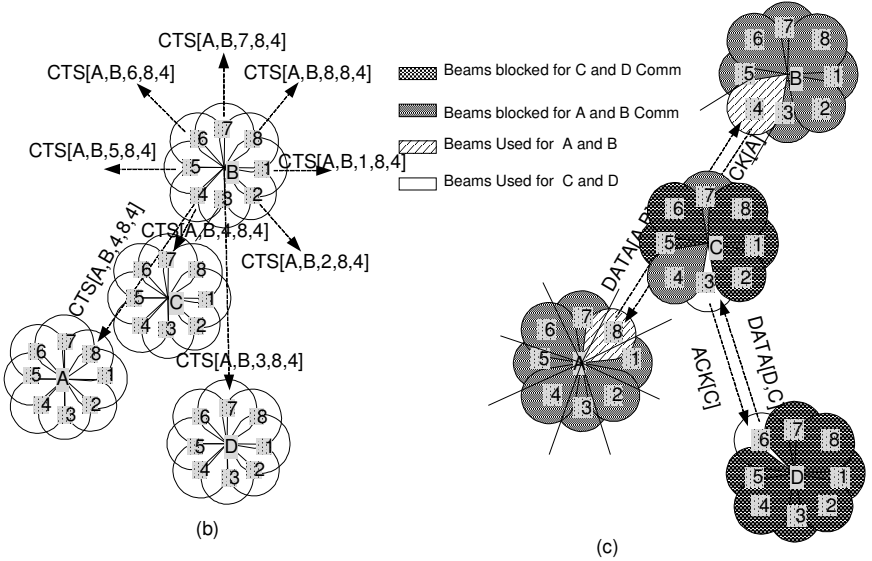

(c)

Figure 5. Beam utilization and signal transmitted for RTS (a) and CTS (b) and DATA and ACK(c)

these beams are on the transmission path of A and B. Node A and B will block their beams except best beam. After receiving CTS from node B, A and B will exchange the DAT and ACK using the beam 8 and 4 of node $\mathrm{A}$ and $\mathrm{B}$ respectively. Meanwhile, if node $\mathrm{D}$ want to communicate to node $\mathrm{C}$, they will exchange the RTS and CTS using the unblocked beams and select the best beam for transmission. After the RTS and CTS exchange, node C will block the remaining beams except beam number 3 and similarly node $\mathrm{D}$ will block all the beams except beam 6 that are selected as best beam for transmission between C and D as depicted in figure. Each node maintains a table that has information of all nodes and their blocked and unblocked beams, best beams at that node.

\section{Performance Evaluation}

\subsection{Simulation Setup}

Simulation is done on Qualnet [21] that is written using PARSEC [22], a C language base discrete event simulator. Traffic model used in our simulation is CBR with packet size of 512 byte and transmission data rate of $2 \mathrm{Mbps}$. The simulations are run for different random seed and the results are statistically averaged out for 25 iterations, each running for 600 simulation seconds. For fair comparison of MAC protocols we have used two different topologies, first is 5 X5 mesh topology with different source and destination combination.

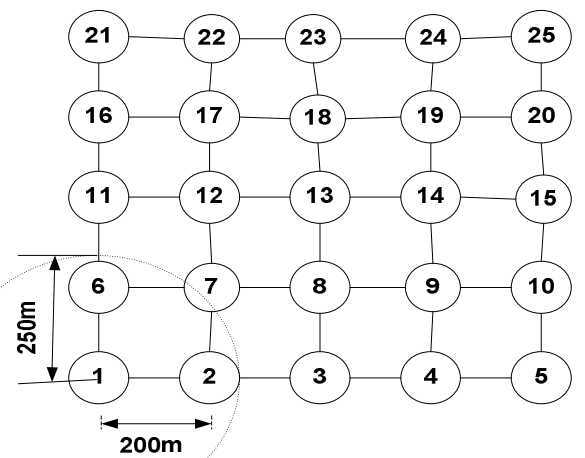

Figure 6. 5X5 mesh network scenario used in simulation 
Row and column are being separated by 200 meters, as given in figure. 6 and table.1. Transmission range of each node is 250 meters. Second topology is random, in which 25 nodes are randomly placed and simulation is done for different source and destination pair for 600 simulation seconds. We have used the AODV (Ad hoc on demand distance vector) routing protocol. Performance of IEEE802.11 MAC, D-MAC, Angular-MAC with eight beams "AMACEB" are compared with performance matrices like throughput.

Table1. Simulation Parameters

\begin{tabular}{|l|l|}
\hline \multicolumn{1}{|c|}{ Parameters } & \multicolumn{1}{c|}{ Values } \\
\hline Topology & Mesh, Random \\
\hline Number of node (mesh , random ) & $5 \mathrm{X} 5,25$ \\
\hline Row spacing (mesh) & 200 meters \\
\hline Column spacing (mesh) & 200 meters \\
\hline Channel frequency & $2.4 \mathrm{GHz}$ \\
\hline Transmission range & $250 \mathrm{~meters}$ \\
\hline Data rate & $2 \mathrm{Mbps}$ \\
\hline Receiving threshold & $-81.0 \mathrm{dBi}$ \\
\hline CS threshold & $-91.0 \mathrm{dBi}$ \\
\hline Main lobe antenna gain & $12.0 \mathrm{dBi}$ \\
\hline Number of beams & 8 \\
\hline Packet size & 512 bytes \\
\hline CBR packet arrival rate & $2 \mathrm{~ms}$ to $600 \mathrm{~ms}$ \\
\hline Simulation time & 600 sec. \\
\hline
\end{tabular}

\subsection{Simulation Results}

In first Scenario we consider the single hop communication between any two node e.g. connection between nodes 1 to $2(1 \rightarrow 2)$ and connection between nodes 6 to $11(6 \rightarrow 11)$. The performance of proposed MAC (Angular-MAC with eight beams "A-MACEB") is higher compared to IEEE802.11 MAC but nearly same as D-MAC and A-MACFB. This is due to fact that, with IEEE802.11 two connections cannot transmits the packets at the same time but using the D-MAC, A-MACEB simultaneous transmission is possible due to multiple beam antenna of each node. Performances of A-MACEB increased with node density, because multiple beam antennas are under utilized in the case of low density but they are efficiently utilized with high node density at the cast of increased processing time. The second scenario used, two multi-hop communication between any node e.g. connection between nodes 1 to $21(1 \rightarrow 21)$ and connection between nodes 1 to $5(1 \rightarrow 5)$. Performance of D-MAC and A-MACEB are better compared to IEEE802.11, but performance of these MAC is nearly equal.

Figure 7 shows the aggregated throughput in third scenario where 10 simultaneous $(1 \rightarrow 5$, $6 \rightarrow 10,11 \rightarrow 15,16 \rightarrow 20,21 \rightarrow 25,1 \rightarrow 21,2 \rightarrow 22,3 \rightarrow 23,4 \rightarrow 24$, and $5 \rightarrow 25$ ) communications are present in network. This illustrates throughput as function of CBR data sending rate for mesh topology. The A-MACEB achieves approximately 40\% improvement in throughput as compared to the $802.11 \mathrm{MAC}$ and $10 \%$ improvement compare to DMAC. In random topology improvement is more compare to mesh topology due to random location of node during simulation this is given in figure 8. Figure 9 and figure 10 show the performance of A-MACEB , DMAC and 802.11MAC for throughput against delay. A-MACEB that gives the performance improvement in both cases but it is better in the random topology. 
International Journal of Wireless \& Mobile Networks (IJWMN) Vol. 3, No. 6, December 2011

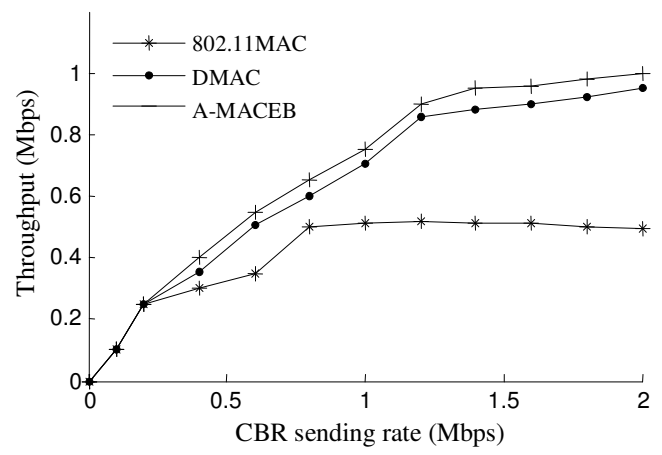

Figure 7. Aggregated throughput comparison of different MAC in mesh topology

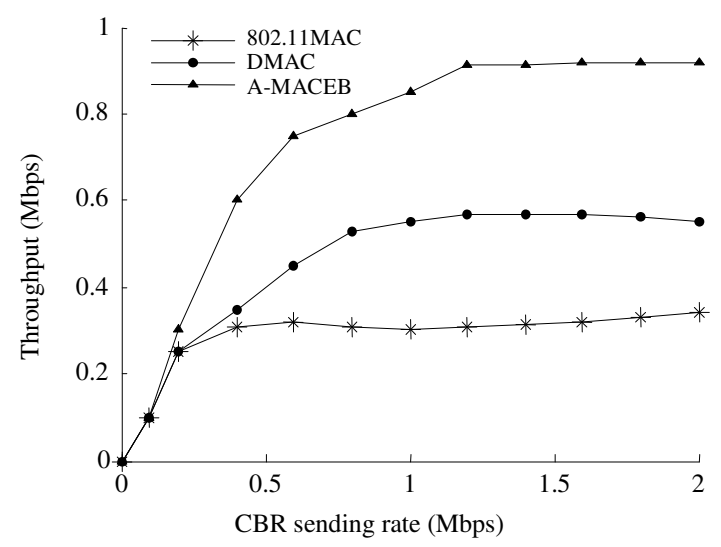

Figure 8. Aggregated throughput comparison of different MAC in random topology

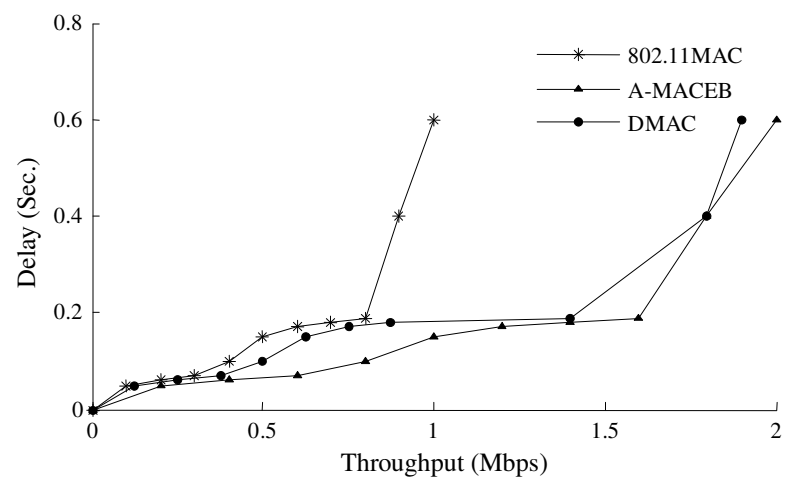

Figure 9. Delay comparison for different throughput in mesh topology 


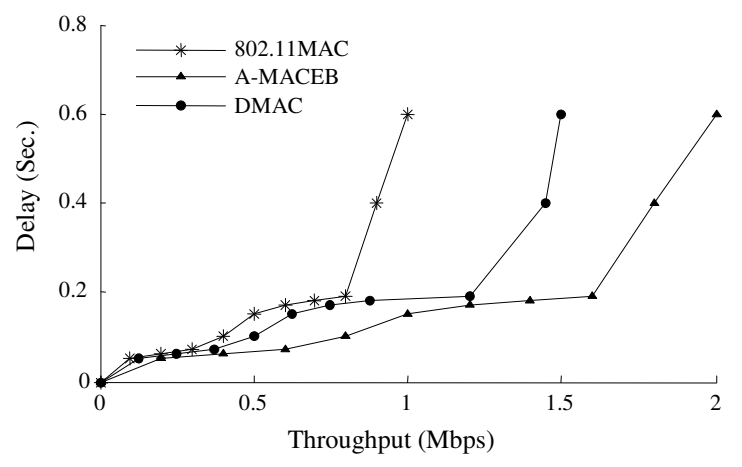

Figure 10. Delay comparison for different throughput in random topology

\section{CONCLuSion}

We have performed comparative analysis of omni-directional and directional MAC for ad-hoc networks. We studied the basic concepts of smart antenna and use of directional antenna in adhoc networks. Further we discuss the MAC protocol in directional antenna and issues like deafness, hidden terminal and HOL problems. We have done simulations to evaluate the performance for CBR traffic on mesh and random topology. The results illustrate that directional MAC protocol A-MACEB and DMAC are more effective than omni-directional MAC 802.11MAC, especially when traffic load is high. It is noticed that A-MACEB achieves the better performance compare to the DMAC.

\section{REFERENCES}

[1] F. A. Tobagi and L. Kleinrock, (1975) "Packet switching in radio channels part II the hidden terminal problem in carrier sense multiple access and busy tone solution", IEEE Transaction on Communication, pp 1417-33.

[2] Phil Karn,(1990) "MACA - A new channel access method for packet radio", in $A R R L / C R R L$ amateur radio $9^{\text {th }}$ computer networking conference, pp 134-140.

[3] V. Bharghavan, Alan Demers, Scott Shenker and Lixia Zhang, (1994)"MACAW: A media access protocol for wireless LAN's”, in ACM SIGCOMM, London, UK, pp 212-125.

[4] "Wireless LAN medium access control (MAC) and physical layer (PHY) specifications", 1997, Draft standard IEEE802.11, P802.11/D1: The editors of IEEE802.11.

[5] G. Li, L.L. Yang, W.S. Conner, B. Sadeghi, (2005) "opportunities and challenges for Mesh Networks Using Directional Antennas”, WiMESH'05, Santa Clara, Califorina, USA.

[6] "Wireless LAN medium access control (MAC) and physical layer (phy) specifications", IEEE standard working group, 1999.

[7] Y.B. Kuo, N.H. Vaidhya, “ Medium access Control protocols using directional antenna in adhoc networks”, Proceeding of IEEE INFOCOM, Tel-Aviv, Israel. March 2000.

[8] R.R. Choudhary, R. Ramanathan, N.H. Vaidhya, (2002)“Using directional antenna for medium access control in adhoc networks", Proceeding of ACM MobiCom, Atlanta, Georgia, USA, pp 23-28.

[9] K. Kobayashi, M. Nakagaw,(2000) “ Spatially Divided channel scheme using sectored Antenna for CSMA/CA Directional CSMA/CA”, Proceeding of IEEE PIMRC, London.

[10] T. Korakis G. Jakallari and L. Tassiulas, (2003)“ A Mac protocol for full exploitation of directional antenna in ad-hoc networks", Proceeding of the $4^{\text {th }}$ ACM International symposium on mobile Ad hoc networking and Computing (MobiHoc), pp 98-107. 
International Journal of Wireless \& Mobile Networks (IJWMN) Vol. 3, No. 6, December 2011

[11] J. Den and Z. Haas,(1998) "Dual busy tone multiple access (DBTMA): A new medium access control for packet radio networks", in ICUPC, Florence, Itally.

[12] Zhuochuan Huang, Chien-Chung Shen, Chavalit Srisathapornphat, and Chaiporn Jaijaeo,(2002)

" A busy-tone based directional MAC protocol for ad-hoc networks," in IEEE MILCOM, Anaheim, CA.

[13] R. R. Choudhury, N. H. Vaidhya, (2004)“Deafness: A mac problem in ad-hoc networks when using directional antennas," Proceeding of the $12^{\text {th }}$ IEEE international Conference on networks Protocols (ICNP), pp. 283-192

[14] Pan Li, Honhqiang Zhai, Yuguang Fang, (2009)“ SDMA: Selective directional MAC protocol for wireless mobile Ad-hoc networks," ACM Transactions on networking, Vol. 7, pp. $1106-$ 1117.

[15] Kai Chen, Fan Jiang, (2007)“A range-adaptive directional MAC protocol for wireless ad hoc networks with smart antennas," International Journal of Electronics and Communications, Volume 61, Issue 10, Pages 645-656.

[16] Z. Huang and C.-C. Shen,(2002) "A Comparison Study of Omni-directional and Directional MAC Protocols for Ad Hoc Networks," Proc. IEEE Globecom.

[17] R. Janaswamy, “ Radio wave propagation and smart antenna for wireless communications," Kluwer Acadmic publisher.

[18] T. Rappaport, (2002) Wireless communications principles and practice, Prentice Hall.

[19] R. Ramanathan, (2001) "On the performance of ad-hoc networks with beam forming antenna", In proceeding of the $2^{\text {nd }} A C M$ international Symposium on Mobile Ad hoc Networking and Computing (MobiHoc), pp 95-105.

[20] E. Ulukan, O. Gurbuz, (2008) "Angular MAC: a frame work for directional antennas in wireless mesh networks", Journal on Wireless Networks, Vol. 14 Issue 2, pp 259-275.

[21] Qualnet Simulator Version 5.0, Scalable Network Technologies,www.scalable-networks.com.

[22] UCLA parallel Computing Laboratory, PARSEC, http://pcl.cs.ucla.edu/, 1999. 